\title{
Long non-coding RNA OECC promotes cell proliferation and metastasis through the PI3K/Akt/mTOR signaling pathway in human lung cancer
}

\author{
YIMIN ZOU $^{1}$, BIN ZHANG ${ }^{1}$, YANXIONG MAO ${ }^{1}$, HAO ZHANG $^{1}$ and WEI HONG ${ }^{2}$ \\ ${ }^{1}$ Department of Respiratory and Critical Care Medicine, The Second Affiliated Hospital, \\ Zhejiang University School of Medicine, Hangzhou, Zhejiang 310009; \\ ${ }^{2}$ Guangzhou Medical University-Guangzhou Institute of Biomedicine and \\ Health Joint School of Life Sciences, Guangzhou Medical University, \\ Guangzhou, Guangdong 510030, P.R. China
}

Received September 7, 2018; Accepted June 24, 2019

DOI: $10.3892 / \mathrm{ol} .2019 .10644$

\begin{abstract}
Lung cancer is one of the most common malignancies worldwide; however, its detailed molecular mechanism remains largely unknown. Long non-coding RNAs (lncRNAs) have been identified to serve critical roles in tumorigenesis. The aim of the present study was to investigate the role of a newly identified lncRNA, overexpressed in colorectal cancer (OECC), in human lung cancer. It was initially revealed that the relative transcript level of OECC was highly upregulated in clinical human lung cancer tissues as well as in cultured lung cancer cells. Knockdown of OECC with specific short hairpin RNAs in lung cancer cell lines A549 and 95D inhibited colony formation and cell viability, as evidenced using colony formation assays and cell proliferation assays. Furthermore, depletion of OECC in A549 and 95D cells suppressed migration and invasion, which was verified using Transwell assays. RNA-sequence analysis suggested that the phosphoinositide 3-kinase/protein kinase B (Akt)/mammalian target of rapamycin signaling pathway was positively regulated by OECC in lung cancer cells A549. In addition, overexpression of Akt in OECC-depleted A549 and 95D cells reversed the suppression of proliferation and migration caused by OECC depletion. The results of the present study identified IncRNA OECC as a novel regulator of lung cancer progression and provided new clues for the clinical treatment of lung cancer.
\end{abstract}

Correspondence to: Dr Wei Hong, Guangzhou Medical University-Guangzhou Institute of Biomedicine and Health Joint School of Life Sciences, Guangzhou Medical University, 195 West Dongfeng Road, Yuexiu, Guangzhou, Guangdong 510030, P.R. China E-mail: weihong5566@sina.com

Key words: overexpressed in colorectal cancer, proliferation, metastasis, phosphoinositide 3-kinase/protein kinase B/mammalian target of rapamycin, lung cancer

\section{Introduction}

Lung cancer is among the most common malignancies in both men and women worldwide (1). The majority of patients with lung cancer are diagnosed when the disease reaches the advanced stages, resulting in a 5-year survival rate of only $3-7 \%$ (2). The poor prognosis makes lung cancer one of the leading causes of cancer-associated mortality, with 1.8 million estimated novel cases and 1.6 million estimated mortalities per year in the United States (3). The majority of patients present with a locally metastatic condition due to the high metastatic potential of lung cancer cells (4). In addition, oncogenic drivers, such as mutations of epidermal growth factor receptor (EGFR), Ki-ras2 Kirsten rat sarcoma viral oncogene homolog, human epidermal growth factor receptor 2 and/or ROS proto-oncogene 1 have also contributed to the development and progression of lung cancer, which means that personalized and genotype-directed therapy, as well as novel immunotherapies (programmed death-1 or programmed death-ligand 1), have revolutionized the management of lung cancer $(5,6)$. It is for this reason that an increasing amount of researchers have been focusing on identifying novel molecular regulators of lung cancer.

Long non-coding RNAs (lncRNAs) are a class of RNAs that consist of $>200$ nucleotides and lack protein-coding abilities $(7,8)$. Previous high-throughput transcriptome analyses have revealed that $>90 \%$ of the genes are transcribed into non-coding RNAs, including IncRNAs, which are predicted to regulate chromatin or function as genetic regulators, depending on their location relative to the nucleus $(9,10)$. To date, $>3,000$ lncRNAs have been identified, $1 \%$ of which only have had their roles identified (9). IncRNAs are suggested to be classified into five categories depending on their origin (11) and have three subtypes based on genomic locations (12), namely intergenic lncRNAs, intronic lncRNAs and antisense IncRNAs.

During recent decades, IncRNAs have been revealed to serve crucial roles in various molecular genetics and cellular processes (13), such as chromosomal dosage compensation, 
maintenance of chromatin structure, splicing, cellular differentiation, cell cycle and tumorigenesis (14). For instance, lncRNA sex determining region Y-box 2 overlapping transcript was reported to regulate cell proliferation and identified as a poor survival indictor in human lung cancer (15). LncRNA SPRY4-IT1 was identified as an emerging factor in tumorigenesis of osteosarcoma (16).

Overexpressed in colorectal cancer (OECC) is a newly identified lncRNA, which originates from chromosome 8q24 and has been revealed as being highly expressed in human colorectal carcinoma (CRC) (17). However, its detailed role and molecular mechanism in other types of tumor remained largely unknown. The aim of the present study was to identify the effects of OECC on cell proliferation and cell metastasis in human lung cancer and sought to uncover the underlying molecular mechanisms. To the best of our knowledge, the present study is the first to investigate the role of OECC in lung cancer, which may provide novel clues for the clinical treatment of lung cancer.

\section{Materials and methods}

Human samples. The present study was approved by the Ethical Committee of Guangzhou Medical University (Guangzhou, China) A total of 50 patients with lung cancer (male/female, 34:16; age range, 59+9 years) were recruited between January 2016 and December 2016 at the First Affiliated Hospital of Guangzhou Medical University and included in the present study. Patients who had received chemotherapy or radiotherapy treatments prior to surgery were excluded from the study. The tumor tissues and adjacent non-tumorous tissues of the patients were dissected during surgery and immediately frozen in liquid nitrogen. Signed informed consent was provided by all patients.

RNA isolation and reverse transcription-quantitative polymerase chain reaction ( $R T-q P C R)$ analysis. Total RNA was extracted from human samples and cultured cells using TRIzol ${ }^{\circledR}$ reagent (Invitrogen; Thermo Fisher Scientific, Inc.) in a volume of $1 \mathrm{ml}$ for each well in 6-well plates. RNAs were quantified using a Nanodrop ${ }^{\mathrm{TM}} 2000$ instrument (Thermo Fisher Scientific, Inc.) according to the manufacturer's protocol. A total of $1 \mu \mathrm{g}$ RNA was transcribed into cDNA using reverse transcriptase (Takara Biotechnology Co., Ltd.) with the following protocols: $37^{\circ} \mathrm{C}$ for $15 \mathrm{~min}$ and $85^{\circ} \mathrm{C}$ for 5 sec. Then, qPCR was performed in an ABI 7900 machine (Applied Biosystems; Thermo Fisher Scientific, Inc.) with hot start Taq DNA polymerase and SYBR ${ }^{\circledR}$ Green (Takara Biotechnology Co., Ltd.). The procedure was as follows: Initial denaturation at $95^{\circ} \mathrm{C}$ for $5 \mathrm{~min}$, followed by 45 repeats of a three-step cycling program consisting of $10 \mathrm{sec}$ at $95^{\circ} \mathrm{C}$ (denaturation), $10 \mathrm{sec}$ at $60^{\circ} \mathrm{C}$ (primer annealing) and $10 \mathrm{sec}$ at $72^{\circ} \mathrm{C}$ (elongation), and a final extension step for $10 \mathrm{~min}$ at $72^{\circ} \mathrm{C}$. The primers used were: OECC forward, $5^{\prime}-\mathrm{AAC}$ CGTAGGAGCACATCACAG-3' and reverse, 5'-CCG TGGTTTCAGTTGCCCTA-3'; phosphoinositide 3-kinase (PI3K) forward, 5'-GTCCTATTGTCGTGCATGTGG-3' and reverse, 5'-TGGGTTCTCCCAATTCAACC-3'; protein kinase B (Akt) forward, 5'-TTCTATGGCGCTGAGATTGTG T-3' and reverse, 5'-GCCGTAGTCATTGTCCTCCAG-3'; mammalian target of rapamycin (mTOR) forward, 5'-ATG CTTGGAACCGGACCTG-3' and reverse, 5'-TCTTGACTC ATCTCTCGGAGTT-3'; and GAPDH forward, 5'-GTGGAC ATCCGCAAAGAC-3' and reverse, 5'-AAAGGGTGTAAC GCAACTA-3'. GAPDH was included as an internal control. The $2^{-\Delta \Delta \mathrm{Cq}}$ method was used to calculate the relative expression normalized to GAPDH (18).

Cell culture and transfection. Normal lung epithelial cell line BEAS-2B and human lung cancer cell lines H1975 and SPC-A-1 were purchased from the American Type Culture Collection (ATCC). Other lung cancer cell lines A549, 95D and H-125 were from the Cell Bank of the Chinese Academy of Sciences. All the cell lines were cultured in Dulbecco's modified Eagle's medium (DMEM; Gibco; Thermo Fisher Scientific, Inc.) supplied with $10 \%$ fetal bovine serum (FBS; Gibco; Thermo Fisher Scientific, Inc.) at $37^{\circ} \mathrm{C}$ with $5 \% \mathrm{CO}_{2}$. The culture medium was replaced once every 2 days, unless otherwise stated. Short hairpin (sh)RNA against OECC (shOECC) were designed by Shanghai GenePharma Co., Ltd. and a negative control shRNA (shNC) was included as a control. Akt-expressing plasmid (pLNCX1 HA Akt1) was purchased from Addgene, Inc. (cat. no. 15990). A total of $1 \times 10^{5}$ cells were transfected with $2 \mu \mathrm{g}$ plasmid using Lipofectamine ${ }^{\circledR} 3000$ (Invitrogen; Thermo Fisher Scientific, Inc.) for $48 \mathrm{~h}$ at $37^{\circ} \mathrm{C}$ according to the manufacturer's protocol.

Colony formation assay. A549 and 95D cells were seeded in 12-well plates in DMEM in triplicate (100 cells/well) and transfected with specific shRNA against OECC. Subsequently, the plates were incubated in a $37^{\circ} \mathrm{C}$ incubator for 14 days and the colonies that contained $>50$ cells were fixed with pre-iced methanol for $10 \mathrm{~min}$ at room temperature and stained with crystal violet $(1 \%)$ for $5 \mathrm{~min}$ at room temperature. Colonies were counted under a light microscope (Nikon Corporation) at a magnification of $\mathrm{x} 200$.

5-Ethynyl-2'-deoxyuridine (EdU) cell proliferation assay. A549 and 95D cells were seeded into 24-well plates and transfected with or without shOECC. At $48 \mathrm{~h}$ post-transfection, medium was replaced with complete DMEM supplemented with $50 \mu \mathrm{M}$ EdU (Thermo Fisher Scientific, Inc.) and further incubated at $37^{\circ} \mathrm{C}$ for $2 \mathrm{~h}$. Following washing with ice-cold PBS twice, the cells were fixed with $4 \%$ polyoxymethylene containing 0.5\% Triton ${ }^{\mathrm{TM}}$ X-100 (Sigma-Aldrich; Merck $\mathrm{KGaA}$ ) for $5 \mathrm{~min}$ at room temperature and then stained with Apollo dye (Thermo Fisher Scientific, Inc.) for $30 \mathrm{~min}$ at $37^{\circ} \mathrm{C}$. Following staining with DAPI $(1: 1,000 ; 10 \mathrm{~min}$ at room temperature), cells were imaged with a light microscope (Nikon Corporation) at a magnification of $\mathrm{x} 200$.

Cell viability assay. A549 and 95D cells were seeded into chamber slides and transfected with shOECC and cultured in a $37^{\circ} \mathrm{C}$ incubator for $48 \mathrm{~h}$ with co-incubation of G418 (Sigma-Aldrich; Merck KGaA). Cell viability was assessed using the Cell Counting Kit-8 (Thermo Fisher Scientific, Inc.) according to the manufacturer's protocol. Relative proliferative rate was assessed by determining the mean and standard deviations of five randomly selected image fields. To evaluate the overall survival rate, a total of $1 \times 10^{3}$ A549 

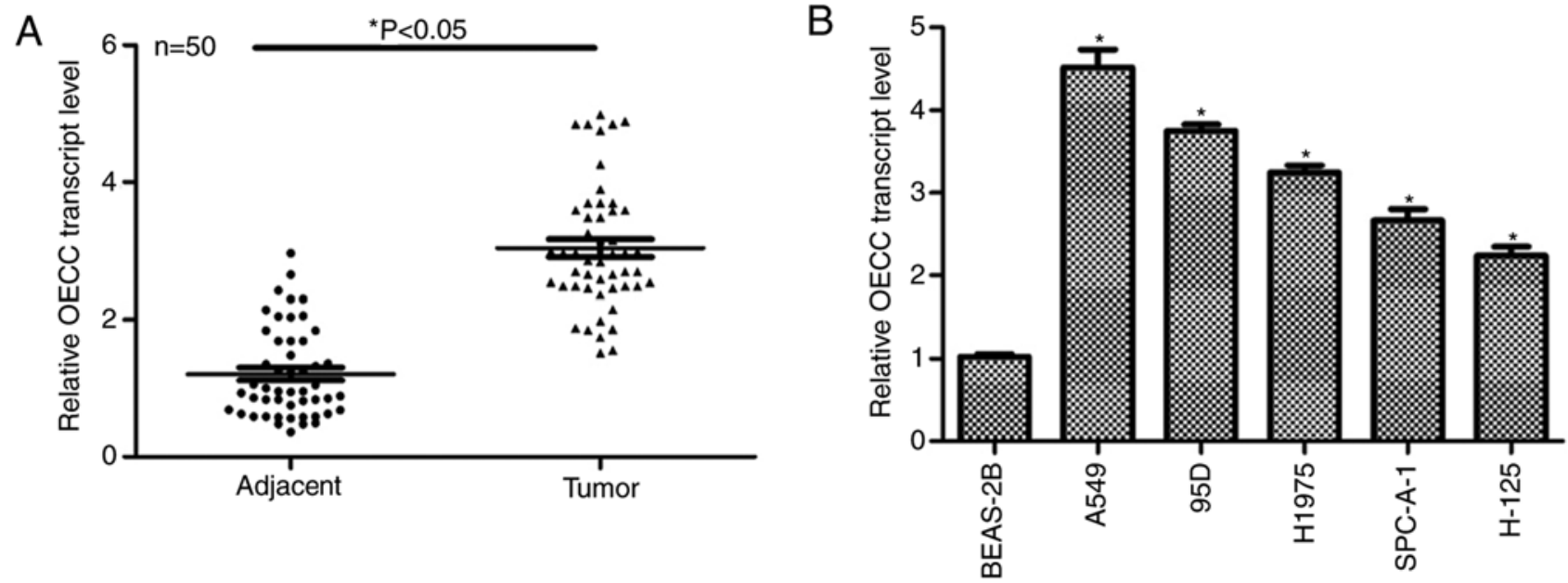

Figure 1. Long non-coding RNA OECC is overexpressed in human lung cancer in vivo and in vitro. (A) The relative transcript level of OECC was determined using RT-qPCR in clinical tissues from patients diagnosed with lung cancer and in adjacent healthy tissues. (B) The relative transcript level of OECC was determined using RT-qPCR in lung cancer cells compared with in normal epithelial cells. " $\mathrm{P}<0.05$, vs. BEAS-2B cells. OECC, overexpressed in colorectal cancer; RT-qPCR, reverse transcription-quantitative polymerase chain reaction.

and 95D cells were seeded into 96-well plates and transfected with shRNAs in triplicate. Following selection for 6 days, overall survival was examined with $0.5 \mathrm{mg} / \mathrm{ml}$ thiazolyl blue tetrazolium blue reagent (Sigma-Aldrich; Merck $\mathrm{KGaA}$ ) and calculated at $590 \mathrm{~nm}$ using a Tecan microplate reader (Tecan Group, Ltd.).

Transwell and Matrigel assay. A549 and 95D were seeded into 6-well plates and transfected with shRNAs against OECC for $72 \mathrm{~h}$. Cells were washed, trypsinized and collected by centrifuge $\left(1,000 \mathrm{x} \mathrm{g}\right.$ for $5 \mathrm{~min}$ at $4^{\circ} \mathrm{C}$. Approximately $1 \times 10^{5}$ cells were seeded into the upper chamber in DMEM without FBS. A volume of $600 \mu \mathrm{l}$ complete DMEM (supplied with 10\% FBS) were added into the lower chamber. The chambers were incubated for another $24 \mathrm{~h}$, fixed with ice-cold methanol for $5 \mathrm{~min}$ at room temperature and stained with crystal violet (1\%) for $5 \mathrm{~min}$ at room temperature. The upper chambers were scraped with a cotton swab and the lower chamber was photographed under a light microscope (Nikon Corporation) with five randomly selected image fields. For the cell invasion assay, the membrane was pre-coated with Matrigel (Corning Inc., Corning, NY, USA) for $6 \mathrm{~h}$ at $37^{\circ} \mathrm{C}$.

RNA sequence analysis. A549 cells were transfected with or without shOECC for $72 \mathrm{~h}$ and the total RNAs were extracted for RNA-sequencing (seq) in triplicate. RNA-seq was performed and analyzed by AnNuo Co. Different signaling pathways were investigated and the various genes $(\mathrm{P}<0.05)$ were classified into corresponding signaling pathways.

Wound-healing assay. A total of $1 \times 10^{5}$ A549 and 95D cells were seeded into 6-well plates and co-incubated with the same amount of shRNAs (shNC or shOECC) for $72 \mathrm{~h}$. Wound-healing assays were performed by creating identical wound areas with $10 \mu 1$ pipette tips for anchorage-dependent A549 and 95D cells. Cells were washed with PBS three times and replaced with fresh, serum-free medium immediately. Images of the cells were captured once the scratch was made $(0 \mathrm{~h})$. After
$24 \mathrm{~h}$ of proliferation, cells were also observed and images were captured under light microscope (Nikon Corporation) at a magnification of $\mathrm{x} 200$ for each group.

Statistical analysis. GraphPad Prism software (version 5.0; GraphPad Software, Inc.) was used for statistical analysis. Independent Student's t-test was used for comparisons between groups, whereas differences between tumor and adjacent normal control samples were analyzed using a paired Student's t-test. For comparisons among multiple groups ( $\geq 3$ groups), one-way analysis of variance was applied, followed by a least significance difference post hoc test. $\mathrm{P}<0.05$ was considered to indicate a statistically significant difference.

\section{Results}

LncRNA OECC is overexpressed in human lung cancer in vivo and in vitro. In total, 50 patients with clinical lung cancer were involved in the present study. Tumor tissues and adjacent non-cancerous tissues were collected from the patients for RT-qPCR analysis. As presented in Fig. 1A, the relative OECC transcript level was markedly increased in tumor tissues compared with in their adjacent non-cancerous counterparts. The expression of OECC was then detected in vitro. As presented in Fig. 1B, the transcript level of OECC was notably upregulated in all the lung cancer cell lines as compared with in the normal lung epithelial cell line BEAS-2B. Notably, A549 and 95D cells exhibited the highest OECC expression of all the lung cancer cell lines. Thus, these two cell lines were chosen for the subsequent functional assays. These data suggested that the transcript level of OECC was upregulated in human lung cancer in vivo and in vitro.

Knockdown of OECC inhibits cell proliferation in A549 and $95 \mathrm{D}$ cells. Next, the expression of OECC was knocked down by specific shOECC in order to investigate the detailed roles of OECC in human lung cancer. As presented in Fig. 2A, three shRNAs against OECC were designed and transfected into 
A

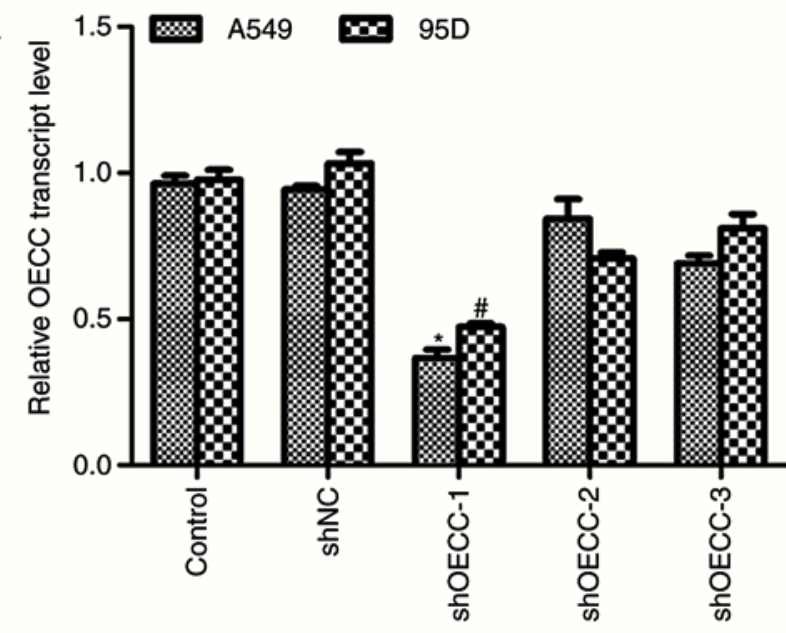

C

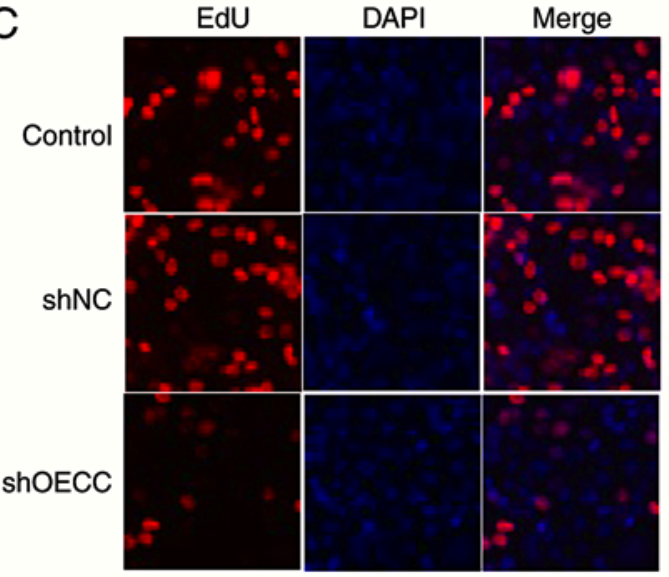

$\mathrm{E}$

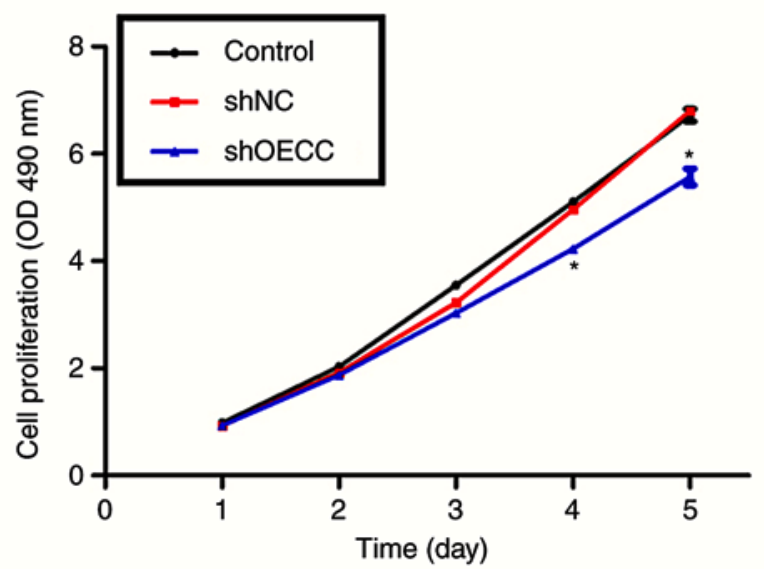

B
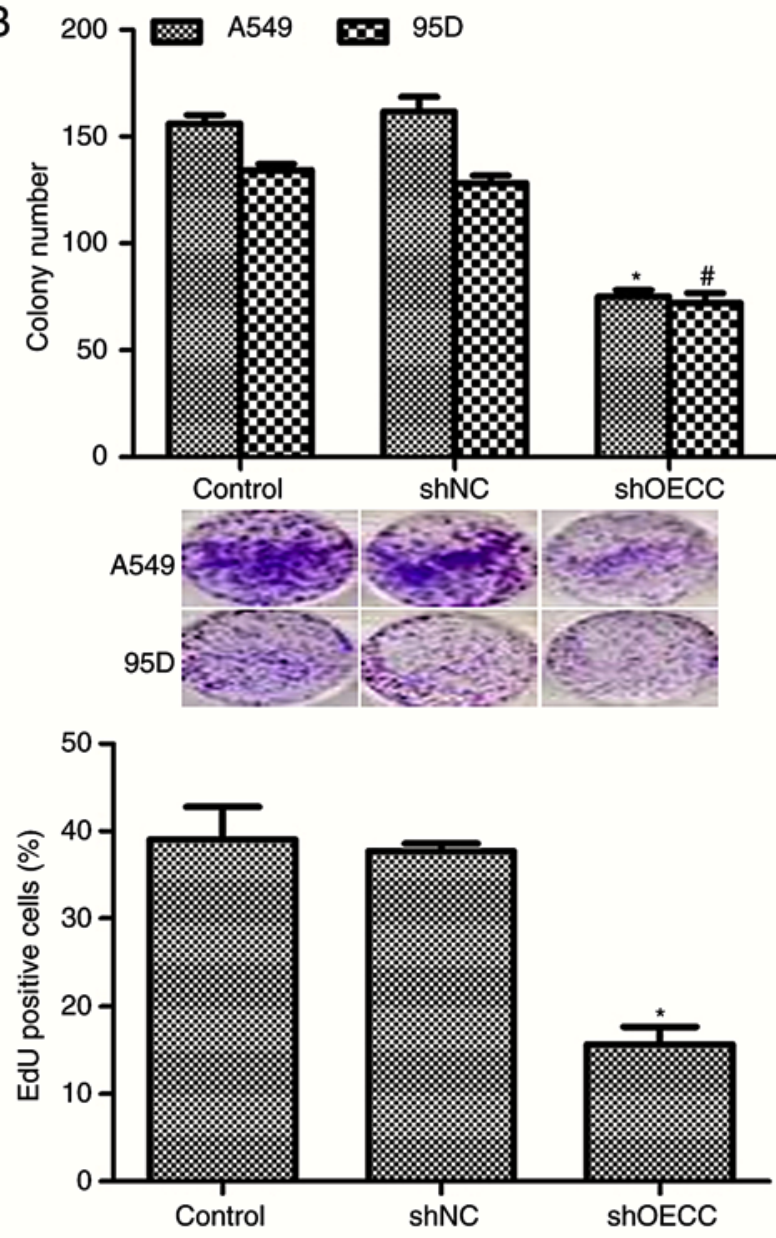

$\mathrm{F}$

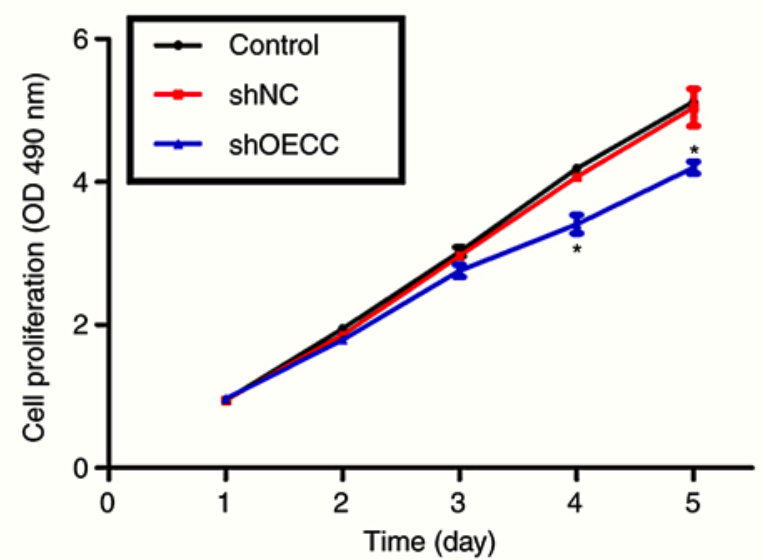

Figure 2. Knockdown of OECC inhibits cell proliferation in A549 and 95D cells. (A) RT-qPCR analysis was performed in A549 and 95D cells transfected with shRNA against OECC. (B) Colony formation assays were performed in A549 and 95D cells transfected with shRNA against OECC. The images included were the representative photos of colony formation assays. ${ }^{*} \mathrm{P}<0.05$, vs. control in A549 cells. " $\mathrm{P}<0.05$, vs. control in $95 \mathrm{D}$ cells. (C) Representative images of EdU assays in A549 cells, (magnification, x200). (D) Quantification of EdU assays in A549 cells transfected with shOECC. (E) Cell proliferation assays were performed in A549 cells over 5 consecutive days when the cells were treated with shOECC. (F) Cell proliferation assays were performed in 95D cells over 5 consecutive days when the cells were treated with shOECC. ${ }^{*} \mathrm{P}<0.05$, vs. control. OECC, overexpressed in colorectal cancer; RT-qPCR, reverse transcription-quantitative polymerase chain reaction; shRNA, short hairpin RNA; EdU, 5-ethynyl-2'-deoxyuridine; shOECC, short hairpin RNA against OECC; OD, optical density; shNC, negative control short hairpin RNA.

A549 and 95D cells and only the first shRNA was effective, thus it was chosen for the subsequent analysis and renamed as shOECC. Colony formation assays revealed $>150$ colonies in the A549 control cells compared with only 75 colonies in
A549 cells following transfection with shOECC. Likewise, transfection of shOECC resulted in the decrease of almost $50 \%$ of $95 \mathrm{D}$ cells (Fig. 2B). The results from the EdU assays, presented in Fig. 2C and D, demonstrated that depletion of 
A
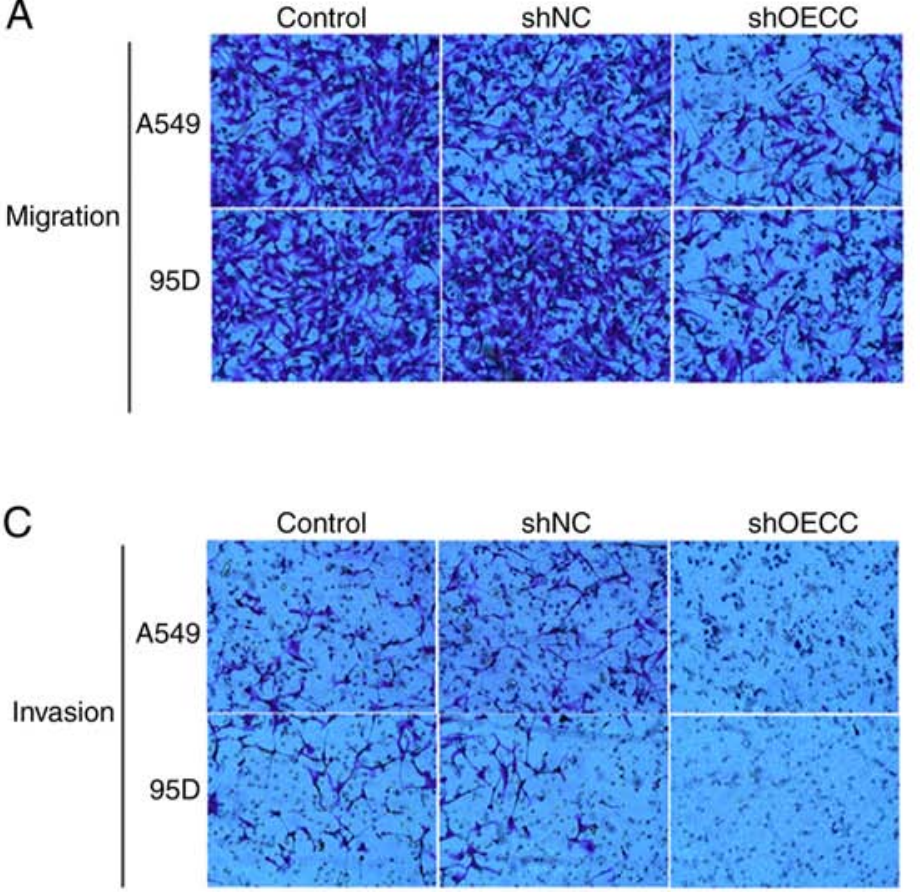

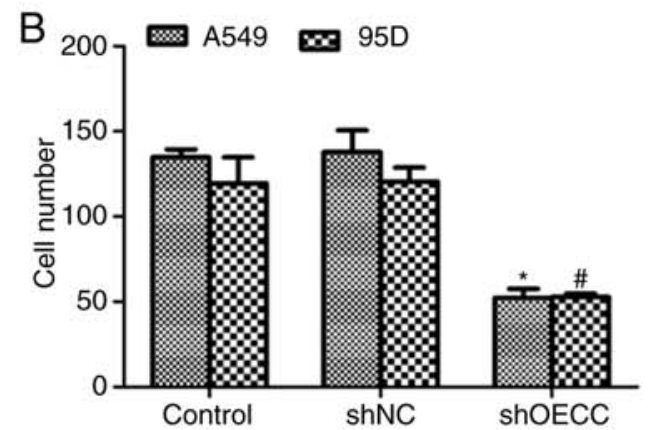

D $\quad$ A549 $\infty 55$

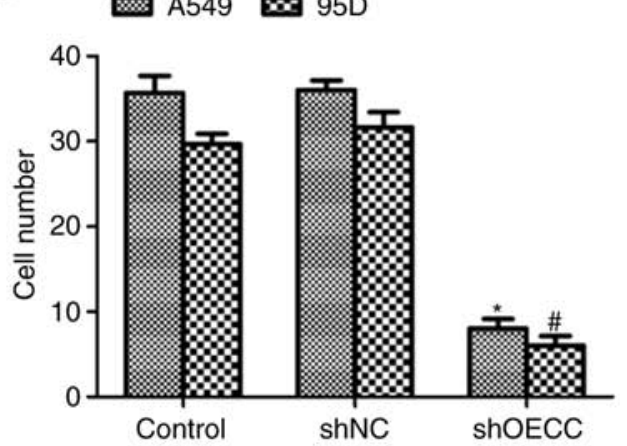

Figure 3. Knockdown of OECC in A549 and 95D cells results in cell metastasis suppression in vitro. (A) Representative images of cell migration assays in A549 and 95D cells transfected with shOECC. (B) Quantification of cell migration assay. (C) Representative images of cell invasion assays in A549 and 95D cells transfected with shOECC. (D) Quantification of cell invasion assay. ${ }^{*} \mathrm{P}<0.05$, vs. control in A549 cells. ${ }^{\#} \mathrm{P}<0.05$, vs. control in $95 \mathrm{D}$ cells. OECC, overexpressed in colorectal cancer; shOECC, short hairpin RNA against OECC; shNC, negative control short hairpin RNA.

OECC in A549 cells resulted in a decrease in EdU positive cells, indicating the effects that OECC suppression has on cell proliferation. Cell viability assays were performed in A549 and 95D cells transfected with shOECC for 5 consecutive days. No clear difference was observed in the first 3 days among the three groups of A549 and 95D cells; however, the rate of cell proliferation was suppressed by almost $20 \%$ on the fourth day and $25 \%$ on the fifth day in A549 cells (Fig. 2E). Similarly, the cell viability of 95D cells was also inhibited by the knockdown of OECC on the fourth and fifth days (Fig. 2F). These data collectively suggested that depletion of OECC in A549 and $95 \mathrm{D}$ cells inhibited cell proliferation in vitro.

Knockdown of OECC in A549 and 95D cells suppresses cell metastasis in vitro. As presented in Fig. $3 \mathrm{~A}$ and $\mathrm{B}$, transfection of shOECC into A549 and 95D cells delayed cell migration through the membrane. In the control group, 35 A549 cells and $\sim 30$ 95D cells invaded the membrane whereas only $\sim 10$ A549 cells and $\sim 89$ 5D cells were observed on the lower surface of the membrane (Fig. 3C and D). These data suggested that depletion of OECC inhibited cell metastasis in human lung cancer cells in vitro.

Knockdown of OECC in A549 cells regulates the PI3K/Akt/mTOR signaling pathway. To elucidate the detailed regulatory molecular mechanism of OECC in cell proliferation and cell metastasis, a RNA-seq analysis was performed. Of all the pathways observed to be altered, the PI3K/Akt/mTOR signaling pathway was the most marked. As presented in Fig. 4, knockdown of OECC in A549 cells decreased the mRNA levels of PI3K, phosphoinositide-dependent kinase-1,
Akt, 5'-AMP-activated protein kinase and endothelial nitric synthase etc., and increased expression of genes such as tumor protein 53, neurofibromin 1 and regulator of cullins-1, which are all part of and/or involved in crosstalk with the $\mathrm{PI} 3 \mathrm{~K} / \mathrm{Akt} / \mathrm{mTOR}$ signaling pathway. These results suggested that OECC expression was closely associated with the $\mathrm{PI} 3 \mathrm{~K} / \mathrm{Akt} / \mathrm{mTOR}$ signaling pathway.

OECC regulates cell proliferation and cell metastasis through the PI3K/Akt/mTOR signaling pathway in human lung cancer. In the present study, the role of the PI3K/Akt/mTOR signaling pathway in the function of OECC in lung cancer was investigated. To this end, Akt-expressing plasmid was co-transfected with shOECC into A549 cells. As presented in Fig. 5A, the mRNA levels of OECC, PI3K, Akt and mTOR were significantly decreased when OECC was knocked down in A549 cells, which was consistent with the result of RNA-seq analysis (Fig. 4). Furthermore, co-incubation of Akt-expressing plasmid increased the Akt and mTOR mRNA levels, but not OECC and PI3K, which hinted that OECC was the upstream regulator of $\mathrm{PI} 3 \mathrm{~K} / \mathrm{Akt} / \mathrm{mTOR}$ signaling. The activated forms of PI3K and Akt were also investigated, namely p-PI3K and p-Akt. Fig. 5B indicates that knockdown of OECC in A549 cells decreased the protein levels of p-PI3K and p-Akt, whereas in contrast, co-overexpression of Akt increased the expression of $\mathrm{p}-\mathrm{PI} 3 \mathrm{~K}$ and p-Akt. Furthermore, knockdown of OECC with specific shRNA inhibited the cells' ability to heal the wound, and in contrast, co-overexpression of Akt increased cell migration abilities in the A549 cells (Fig. 5C and E) and the 95D cells (Fig. 5D and E). Subsequently, depletion of OECC inhibited the formation of colonies in A549 and 95D cells, but co-treatment 


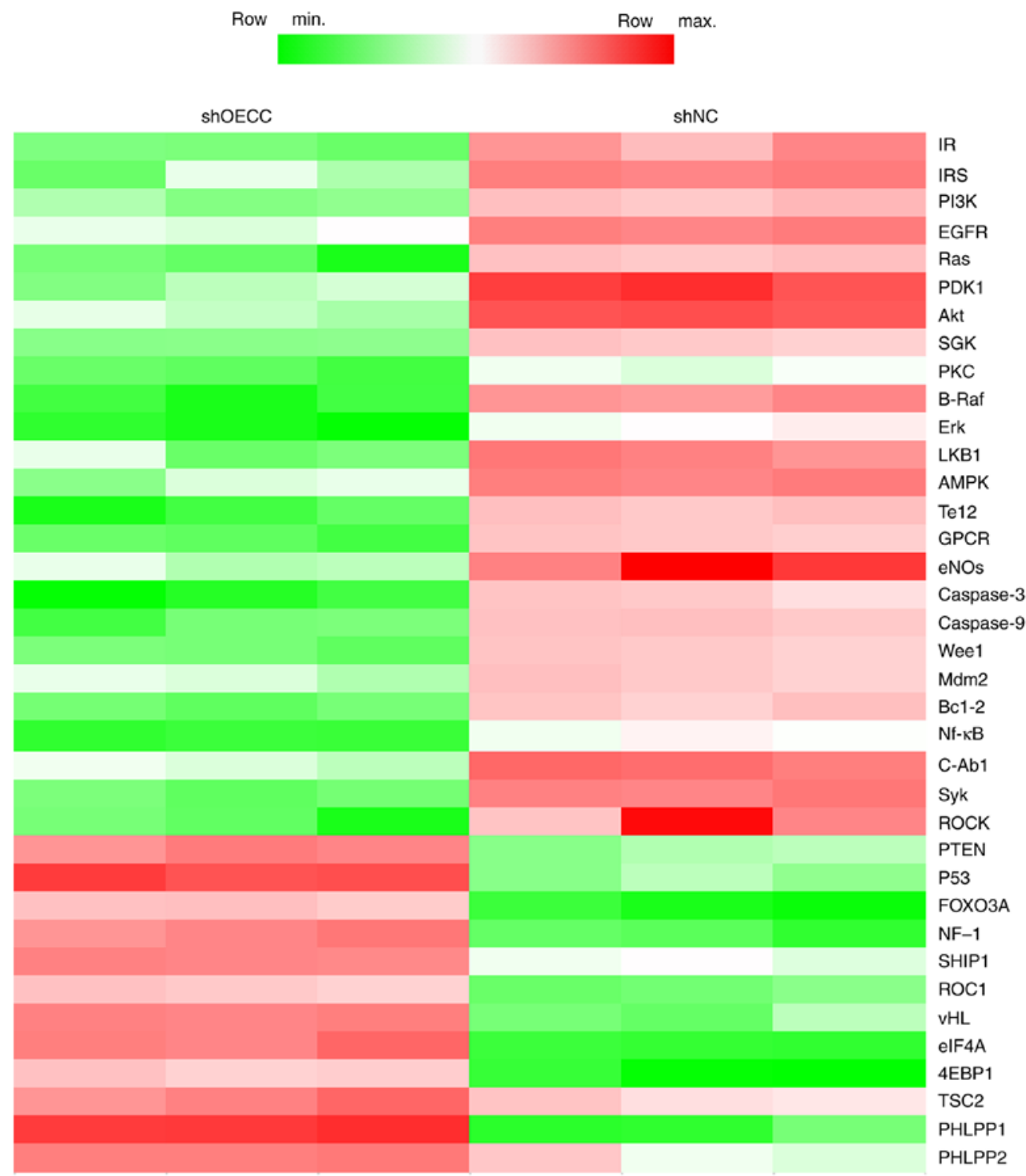

Figure 4. Knockdown of OECC in A549 cells downregulates the PI3K/Akt/mTOR signaling pathway. A549 cells in the presence or absence of shOECC were collected in triplicate and applied for RNA-sequencing analysis. The heat map revealed the gene expression in PI3K/Akt/mTOR signaling pathway. OECC, overexpressed in colorectal cancer; PI3K, phosphoinositide 3-kinase; Akt, protein kinase-B; mTOR, mammalian target of rapamycin; shOECC, short hairpin RNA against OECC; shNC, negative control short hairpin RNA.

with Akt-expressing plasmids reversed these effects (Fig. 5F). These data suggested that OECC regulated cell proliferation and cell metastasis through the PI3K/Akt/mTOR signaling pathway.

\section{Discussion}

Lung cancer remains a marked threat to human health. The overall survival rate continues to be low despite efforts to make improvements over the last few decades (19). Recently, studies have revealed that aberrant transcript levels of lncRNA is involved in the tumorigenesis and tumor progression of human lung cancer (19-21). Of note, there are multiple genes located on chromosome q824 that increase the risk of developing lung cancer, such as CCAT1 (20-22), which promoted cell metastasis via epithelial-to-mesenchymal transition in lung adenocarcinoma (20) and the Wnt signaling in non-small cell lung cancer (21). The present study also indicated that the novel
lncRNA OECC, located on chromosome band q 824 , upregulated cell proliferation and metastasis in human lung cancer. However, the limitations of the present study are that the subtype of lung cancer tissues was not determined, and the correlation between OECC expression and EGFR inhibitors was not investigated; these issues will form the basis of future studies.

LncRNA OECC was first identified by Huang et al (17) in human CRC, where OECC was revealed to be overexpressed in human CRC tissues and cultured CRC cells. Attenuation of OECC inhibited CRC cell proliferation and metastasis, and increased cell apoptosis, since OECC was demonstrated to be a direct target of microRNA-143-3p, leading to the downregulation of its target genes, including nuclear factor- $\mathrm{\kappa B}$ and p38 mitogen-activated protein kinase signaling pathways (17). In the present study, OECC was also demonstrated to be upregulated in lung cancer and associated with cell proliferation and metastasis. Furthermore, with the aid of the RNA-seq technique, it was identified that OECC regulated lung cancer 

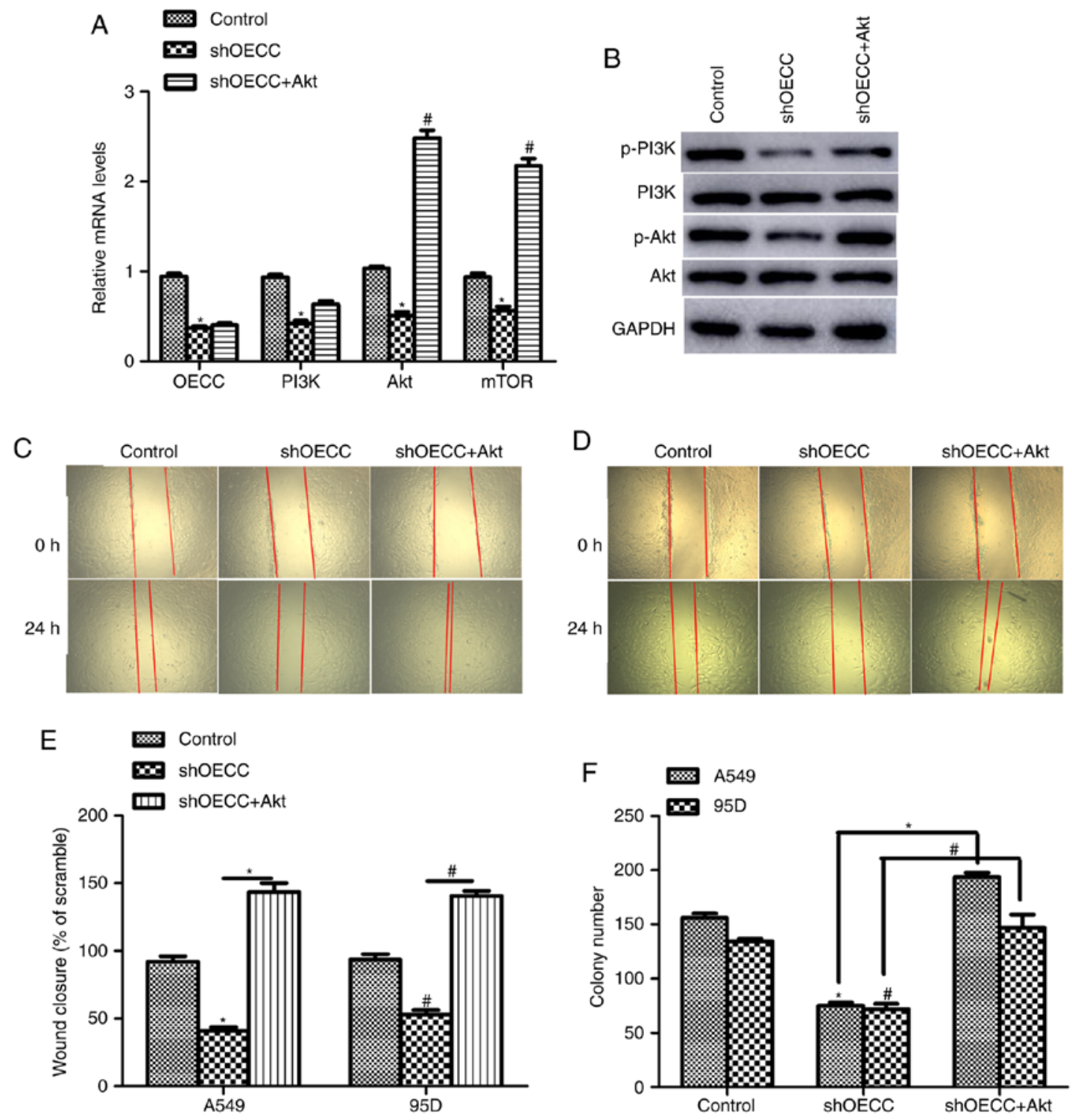

Figure 5. OECC regulates cell proliferation and cell metastasis through the PI3K/Akt/mTOR signaling pathway in human lung cancer. (A) The relative mRNA levels of OECC, PI3K, Akt and mTOR in A549 cells transfected with shOECC in the presence or absence of Akt-expressing plasmid. "P $<0.05$, shOECC vs. control. " $\mathrm{P}<0.05$, shOECC+Akt vs. shOECC. (B) Western blot assays were performed in A549 cells treated with shOECC. (C) Representative images of wound-healing assays in A549 cells. (D) Representative images of wound-healing assays in 95D cells. (E) Quantification assay of wound-healing assay in A549 and 95D cells transfected with shOECC in the presence or absence of Akt-expressing plasmid. " $\mathrm{P}<0.05$, shOECC vs. control. "P<0.05, shOECC+Akt vs. shOECC. (F) Colony formation assays were used in both A549 and 95D cells transfected with shOECC in the presence or absence of Akt-expressing plasmid. ${ }^{*} \mathrm{P}<0.05$, vs. control or as indicated in A549 cells. ${ }^{*} \mathrm{P}<0.05$, vs. control or as indicated in 95D cells. PI3K, phosphoinositide 3-kinase; Akt, protein kinase-B; mTOR, mammalian target of rapamycin; p, phosphorylated; shOECC, short hairpin RNA against OECC; shNC, negative control short hairpin RNA.

progression through PI3K/Akt/mTOR signaling. Thousands of genes were identified to be altered when A549 cells were transfected with shOECC; the present study classified these genes into associated pathways and revealed that the genes in the PI3K/Akt/mTOR signaling pathway were the most marked and, thus, this pathway was investigated further. Comparing with the aforementioned study (17), depletion of OECC in $\mathrm{CRC}$ and lung cancer resulted in similar phenotypes; however, the underlying molecular mechanism was markedly different.

$\mathrm{PI} 3 \mathrm{~K} / \mathrm{Akt} / \mathrm{mTOR}$ signaling is an intracellular pathway that regulates the cell cycle, making it directly associated with cell proliferation, cellular quiescence, cancer and longevity (23). Activation of PI3K phosphorylates and activates Akt, resulting in its translocation onto the plasma membrane and activate multiple downstream effects, including the inhibition of p27 (24) and activation of cAMP response element-binding protein and mTOR (25). The present study revealed that the downregulation of OECC decreased the mRNA levels of PI3K, Akt and mTOR, and inhibited signaling of the whole pathway. Thus, PI3K/Akt/mTOR signaling was activated when OECC was depleted in A549 and 95D cells. Since a PI3K-expressing plasmid could not be commercially purchased, Akt was overexpressed as an alternative, using its expression plasmid to activate this signaling. It was revealed that overexpression of Akt significantly blunted the suppression effects of OECC on cell proliferation and cell metastasis, which reinforced the conclusion that OECC regulated lung cancer progression through the PI3K/Akt/mTOR signaling pathway. However, the detailed molecular mechanism underlying how OECC regulates the PI3K/Akt/mTOR signaling pathway remains 
unknown. The PI3K regulatory subunit has two major isoforms, p85 $\alpha$ and P85 $\beta$, which has been identified as being regulated by a newly identified lncRNA, AK023948 (26), which has been demonstrated to interact with multiple DNAs, RNAs and proteins, which may provide hints for investigating the regulatory details of the PI3K/Akt/mTOR signaling by OECC. Indeed, the present study is just a preliminary investigation of the role of OECC in human lung cancer and, thus, further mechanistic studies should be performed in order to fully corroborate the role of OECC in human lung cancer.

In conclusion, the present study revealed that the recently discovered oncogenic lncRNA, OECC, is also involved in human lung cancer. Depletion of lncRNA OECC suppressed cell proliferation and metastasis in A549 and 95D cells. These results may provide novel clues for the treatment of lung cancer in the clinical setting.

\section{Acknowledgements}

Not applicable.

\section{Funding}

No funding was received.

\section{Availability of data and materials}

The datasets used and/or analyzed during the present study are available from the corresponding author upon reasonable request.

\section{Authors' contributions}

$\mathrm{YZ}$ and $\mathrm{BZ}$ performed the experiments. YM and $\mathrm{HZ}$ helped analyzing data and revised the manuscript. WH designed the project and prepared the manuscript. All authors read and approved the final manuscript.

\section{Ethics approval and consent to participate}

The present study was approved by the Ethical Committee of Guangzhou Medical University (Guangzhou, China). Signed informed consent was provided by all patients.

\section{Patient consent for publication}

Not applicable.

\section{Competing interests}

The authors declare that they have no competing interests.

\section{References}

1. Wang T, Nelson RA, Bogardus A and Grannis FW Jr: Five-year lung cancer survival: Which advanced stage nonsmall cell lung cancer patients attain long-term survival? Cancer 116: 1518-1525, 2010.

2. Tracey E, Roder D, Zorbas H, Villanueva E, Jelfs P and Bishop J: Survival and degree of spread for female breast cancers in New South Wales from 1980 to 2003: Implications for cancer control. Cancer Causes Control 19: 1121-1130, 2008.

3. Levy A and Doyen J: Metformin for non-small cell lung cancer patients: Opportunities and pitfalls. Crit Rev Oncol Hematol 125: 41-47, 2018.
4. DeSantis CE, Siegel RL, Sauer AG, Miller KD, Fedewa SA, Alcaraz KI and Jemal A: Cancer statistics for African Americans, 2016: Progress and opportunities in reducing racial disparities. CA Cancer J Clin 66: 290-308, 2016.

5. Herbst RS, Baas P, Kim DW, Felip E, Pérez-Gracia JL, Han JY Molina J, Kim JH, Arvis CD, Ahn MJ, et al: Pembrolizumab versus docetaxel for previously treated,PD-L1-positive, advanced non-small-cell lung cancer (KEYNOTE-010): A randomised controlled trial. Lancet 387: 1540-50, 2016.

6. Solomon BJ, Mok T, Kim DW, Wu YL, Nakagawa K, Mekhail T, Felip E, Cappuzzo F, Paolini J, Usari T, et al: First-line crizotinib versus chemotherapy in ALK-positive lung cancer. N Engl J Med 371: 2167-2177, 2014

7. Spizzo R, Almeida MI, Colombatti A and Calin GA: Long non-coding RNAs and cancer: A new frontier of translational research? Oncogene 31: 4577-4587, 2012.

8. Chu C, Spitale RC and Chang HY: Technologies to probe functions and mechanisms of long noncoding RNAs. Nat Struct Mol Biol 22: 29-35, 2015.

9. Hutchinson JN, Ensminger AW, Clemson CM, Lynch CR, Lawrence JB and Chess A: A screen for nuclear transcripts identifies two linked noncoding RNAs associated with SC35 splicing domains. BMC Genomics 8: 39, 2007.

10. Yunusov D, Anderson L, DaSilva LF, Wysocka J, Ezashi T, Roberts RM and Verjovski-Almeida S: HIPSTR and thousands of lncRNAs are heterogeneously expressed in human embryos, primordial germ cells and stable cell lines. Sci Rep 6: 32753, 2016.

11. Ponting CP, Oliver PL and Reik W: Evolution and functions of long noncoding RNAs. Cell 136: 629-641, 2009.

12. Wang KC and Chang HY: Molecular mechanisms of long noncoding RNAs. Mol Cell 43: 904-914, 2011.

13. Clark MB and Mattick JS: Long noncoding RNAs in cell biology. Semin Cell Dev Biol 22: 366-376, 2011.

14. Chen J, Wang R, Zhang K and Chen LB: Long non-coding RNAs in non-small cell lung cancer as biomarkers and therapeutic targets. J Cell Mol Med 18: 2425-2436, 2014.

15. Hou Z, Zhao W, Zhou J, Shen L, Zhan P, Xu C, Chang C, Bi H, Zou J, Yao X, et al: A long noncoding RNA Sox 2ot regulates lung cancer cell proliferation and is a prognostic indicator of poor survival. Int J Biochem Cell Biol 53: 380-388, 2014.

16. Li Z, Shen J, Chan MTV and Wu WKK: The long non-coding RNA SPRY4-IT1: An emerging player in tumorigenesis and osteosarcoma. Cell Prolif 51: e12446, 2018.

17. Huang F, Wen C, Zhuansun Y, Huang L, Chen W, Yang X and Liu H: A novel long noncoding RNA OECC promotes colorectal cancer development and is negatively regulated by miR-143-3p. Biochem Biophys Res Commun 503: 2949-2955, 2018.

18. Livak KJ and Schmittgen TD: Analysis of relative gene expression data using real-time quantitative PCR and the 2(-Delta Delta C(T)) method. Methods 25: 402-408, 2001.

19. Mari-Alexandre J,Diaz-Lagares A,Villalba M,Juan O,Crujeiras AB, Calvo A and Sandoval J: Translating cancer epigenomics into the clinic: Focus on lung cancer. Transl Res 189: 76-92, 2017.

20. Lin H, Cheng W, Yan $\mathrm{H}$ and Zhang $\mathrm{X}$ : Overexpression of the long noncoding RNA CCAT1 promotes metastasis via epithelial-to-mesenchymal transition in lung adenocarcinoma. Oncol Lett 16: 1809-1814, 2018.

21. Xu C, Xiao G, Zhang B, Wang M, Wang J, Liu D, Zhang J, Ren $\mathrm{H}$ and Sun X: CCAT1 stimulation of the symmetric division of NSCLC stem cells through activation of the Wnt signalling cascade. Gene Ther 25: 4-12, 2018.

22. Hu B, Zhang H, Wang Z, Zhang F, Wei H and Li L: LncRNA CCAT1/miR-130a-3p axis increases cisplatin resistance in non-small-cell lung cancer cell line by targeting SOX4. Cancer Biol Ther 18: 974-983, 2017.

23. King D, Yeomanson D and Bryant HE: PI3King the lock: Targeting the PI3K/Akt/mTOR pathway as a novel therapeutic strategy in neuroblastoma. J Pediatr Hematol Oncol 37: 245-251, 2015.

24. Rafalski VA and Brunet A: Energy metabolism in adult neural stem cell fate. Prog Neurobiol 93: 182-203, 2011.

25. Man HY, Wang Q, Lu WY, Ju W, Ahmadian G, Liu L, D'Souza S, Wong TP, Taghibiglou C, Lu J, et al: Activation of PI3-kinase is required for AMPA receptor insertion during LTP of mEPSCs in cultured hippocampal neurons. Neuron 38: 611-624, 2003.

26. Koirala P, Huang J, Ho TT, Wu F, Ding X and Mo YY: LncRNA AK023948 is a positive regulator of AKT. Nat Commun 8: 14422, 2017. 